\title{
Human GPRC6A Mediates Testosterone-Induced Mitogen- Activated Protein Kinases and mTORC1 Signaling in Prostate Cancer Cells $\mathbf{s}$
}

\author{
Ruisong Ye, Min Pi, Mohammed M. Nooh, Suleiman W. Bahout, and L. Darryl Quarles \\ Departments of Medicine (R.Y., M.P., L.D.Q.) and Pharmacology (S.W.B.), University of Tennessee Health Science Center, \\ Memphis, Tennessee; and Department of Biochemistry, Faculty of Pharmacy, Cairo University, Cairo, Egypt (M.M.N.)
}

Received October 24, 2018; accepted March 6, 2019

\section{ABSTRACT}

G protein-coupled receptor family $\mathrm{C}$ group 6 member A (GPRC6A) is activated by testosterone and modulates prostate cancer progression. Most humans have a GPRC6A variant that contains a recently evolved $\mathrm{KGKY}$ insertion/deletion in the third intracellular

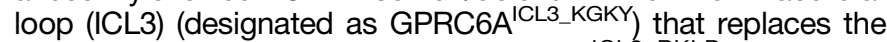

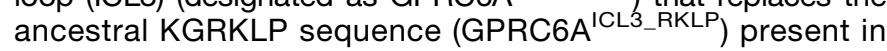
all other species. In vitro assays purport that human GPRC6A ${ }^{\text {ICL3_KGKY }}$ is retained intracellularly and lacks function. These findings contrast with ligand-dependent activation and coupling to mammalian target of rapamycin complex 1 (mTORC1)

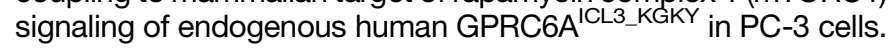
To understand these discrepant results, we expressed mouse (mGPRC6A $A^{\text {ICL3_KGRKLP), human (hGPRC6A }} A^{\text {ICL3_KGKY }}$, and humanized mouse (mGPRC6A $A^{\text {ICL3_KGKY) }}$ GPRC6A into human embryonic kidney 293 cells. Our results demonstrate that mGPRC6A ${ }^{\text {ICL3_KGRKLP }}$ acts as a classic G protein-coupled receptor, which is expressed at the cell membrane and internalizes in response to ligand activation by testosterone. In contrast,
hGPRC6A ${ }^{\text {ICL3_KGKY }}$ and humanized mouse mGPRC6A ${ }^{\text {ICL3_KGKY }}$ are retained intracellularly in ligand naive cells, yet exhibit $\beta$-arrestin-dependent signaling responses, mitogen-activated protein kinase [i.e., extracellular signal-regulated kinase (ERK)], and p70S6 kinase phosphorylation in response to testosterone,

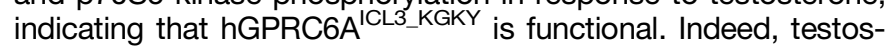
terone stimulates time- and dose-dependent activation of ERK, protein kinase $\mathrm{B}$, and $\mathrm{mTORC} 1$ signaling in wild-type PC-3 cells that express endogenous GPRC6A $A^{\text {ICL3_KGKY }}$. In addition, testosterone stimulates GPRC6A-dependent cell proliferation in wildtype PC-3 cells and inhibits autophagy by activating mTORC1 effectors eukaryotic translation initiation factor $4 \mathrm{E}$ binding protein 1 and Unc-51 like autophagy activating kinase 1. Testosterone activation of GPRC6A has the obligate requirement for calcium in the incubation media. In contrast, in GPRC6A-deficient cells, the effect of testosterone to activate downstream signaling is abolished, indicating that human GPRC6A is required for mediating the effects of testosterone on cell proliferation and autophagy.

\section{Introduction}

G protein-coupled receptor family $\mathrm{C}$ group 6 member A (GPRC6A) is a member of the class-C G protein-coupled receptor (GPCR) family that is widely expressed and activated by multiple ligands, including the peptide osteocalcin (Ocn), cations, basic amino acids, and testosterone (Pi et al., 2016b). The linkage of a Venus flytrap (VFT) motif to the classic GPCR 7-transmembrane domain accounts for the ligand diversity of GPRC6A (Pi et al., 2012, 2015, 2018a). Mouse GPRC6A is proposed to regulate complex endocrine networks and metabolic processes, as demonstrated in in vitro cell culture and

Funding was provided by the National Institutes of Health [Grant R01AR37308] and the American Diabetes Association [Grant 1-13-BS-149-BR (to L.D.Q.)].

The authors declare that they have no conflicts of interest with the contents of this article.

https://doi.org/10.1124/mol.118.115014.

S This article has supplemental material available at molpharm.aspetjournals.org. in vivo animal models (Ferron et al., 2010; Karsenty and Olson, 2016; Pi et al., 2016a,b). Genetic loss-of-function studies and pharmacological activation of GPRC6A in mice show that GPRC6A regulates insulin secretion and proliferation by $\beta$-cells (Pi et al., 2011, 2012; Oury et al., 2013; Wei et al., 2014), glucose uptake and interleukin 6 secretion by skeletal muscle (Mera et al., 2016), testosterone secretion by testicular Leydig cells (De Toni et al., 2014), glucose and fat metabolism by hepatocytes and adipocytes (Otani et al., 2015), and prostate cancer progression ( $\mathrm{Pi}$ and Quarles, 2012). GPRC6A in these tissues senses Ocn released from bone to create endocrine networks regulating energy homeostasis and sexual reproduction (Pi et al., 2016b). GPRC6A also mediates the rapid, nongenomic effects of testosterone in peripheral tissues, including Leydig cells, $\beta$-cells, skeletal muscle, and skin keratinocytes (Pi et al., 2010, 2015; Ko et al., 2014).

ABBREVIATIONS: 4E-BP1, eukaryotic translation initiation factor 4E binding protein 1; AKT, protein kinase B; AR, androgen receptor; ERK, extracellular signal-regulated kinase; GAPDH, glyceraldehyde 3-phosphate; GPCR, G protein-coupled receptor; GPRC6A, G protein-coupled receptor family C group 6 member A; HEK, human embryonic kidney; ICL3, third intracellular loop; KO, knockout; LC3, autophagy marker light chain 3; MK2206, 8-[4-(1-aminocyclobutyl)phenyl]-9-phenyl-2H-[1,2,4]triazolo[3,4-f][1,6]naphthyridin-3-one; mTOR, mammalian target of rapamycin; mTORC1, mTOR complex 1; Ocn, osteocalcin; S6, ribosomal protein S6; S6K, p70S6 kinase; VFT, Venus flytrap; WT, wild type. 
A KGKY polymorphism in the third intracellular loop (ICL3) of GPRC6A evolved in most humans (GPRC6 ${ }^{\text {ICL3_KGKY) }}$ (Jørgensen et al., 2017) to replace the ancestral KGRKLP (GPRC6A ${ }^{\text {ICL3_KGRKLP }}$, rs386705086) sequence present in all animal species. Human embryonic kidney (HEK) 293 cells transfected with mouse GPRC6 $\mathrm{A}^{\mathrm{ICL} \_ \text {KGRKLP }}$ show that this ancestral variant localizes to the cell surface membrane, and imparts signaling responses to Ocn, testosterone, L-Arginine, and cations (Pi et al., 2005, 2010, 2012, 2015, 2016a, 2018a; Oury et al., 2013; Mera et al., 2016; Ye et al., 2017). In contrast,

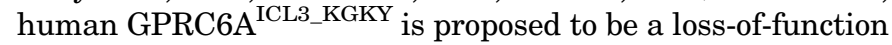
polymorphism that is not associated with any human diseases (Jørgensen et al., 2017). Studies of a human cDNA transfected into HEK-293 cells found that the GPRC6A ${ }^{\text {ICL3_KGKY }}$ variant is retained intracellularly and lacks responsiveness to Ocn and testosterone (Jacobsen et al., 2017; Jørgensen et al., 2017). If this scenario is correct, a functional GPRC6A in humans would be limited to the $20 \%$ of Caucasians and $40 \%$ of people of African decent that express the ancestral KGRKLP polymorphism (Pi et al., 2016b).

In the current study, we determined the distribution of transfected mouse, human GPRC6A, and humanized mouse GPRC6A ${ }^{\mathrm{ICL} 3}$ _KGKY constructs in naive and testosteronestimulated HEK-293 cells. We also examined the function effects of GPRC6A ligands in wild-type (WT) PC-3 cells expressing endogenous GPRC6 $\mathrm{A}^{\mathrm{ICL} 3 \text { KGKKY }}$ and in cells after ablation of GPRC6A. We found that GPRC6A $\mathrm{A}^{\mathrm{ICL} 3 \text { KGRKLP }}$ is cell surface expressed, imparts second messenger signaling, and undergoes internalization in response to GPRC6A agonists, consistent with a classic GPCR. In contrast, we confirmed that GPRC6 $\mathrm{A}^{\mathrm{ICL} 3 \text { KGKY }}$ is retained intracellularly, but leads to mitogen-activated protein kinase [i.e., extracellular signal-regulated kinase (ERK)] and mTORC1 activation in response to ligands. Thus, the GPRC6 $\mathrm{A}^{\mathrm{ICL} 3 \text { KGGKY }}$ polymorphism is not a loss-of-function polymorphism but may have evolved to link to nutrient sensing to ERK and mTORC1 pathways after internalization to endosomes.

\section{Materials and Methods}

Establishment of CRISPR/Cas9-Mediated GPRC6A Knockout Cell Clones. In the present study, one GPRC6A knockout (KO) single clone B12 was selected from our previously established CRISPR/Cas9-edited GPRC6A PC-3 cell line (Ye et al., 2017). We obtained a total of 11 single clones using a serial dilution method, in which B12 contained the 2nt insert (the sequence was confirmed by T-A cloning sequencing) and caused frameshift mutation and loss of function in response to ligand stimulation (for details, see Supplemental Figs. 1 and 2).

Cell Culture and Treatment. Cells were cultured in RPMI 1640 media and cell proliferation assays as previously described (Ye et al., 2017). Briefly, $4 \times 10^{3}$ cells were seeded in the each well of a 96 -well plate, with or without $50 \mathrm{nM}$ testosterone, and the 3-(4,5-Dimethylthiazol-2-yl)-2,5-Diphenyltetrazolium Bromide dye production (MTT) method was used to measure for cell proliferation (Cayman Chemical). For treatment, cells were placed in Hanks' balanced salt solution (Thermo Fisher Scientific), supplemented with $0.5 \mathrm{mM} \mathrm{Ca}^{2+}$ if not otherwise stated, for 2 to 3 hours before stimulation. For autophagy assay, we used GPRC6A KO (B12) and GPRC6A WT control cells (with Cas9 expression and without short guide RNA expression) to examine autophagy marker light chain 3 (LC3) B level and phosphorylation of Unc-51 like autophagy activating kinase 1 . Cell autophagy is induced by 5 -hour starvation in Hanks' balanced salt solution with $50 \mu \mathrm{M}$ chloroquine (Lee et al.,
2007). In addition, both GPRC6A KO cells and control cells were transfected with RFP-LC3 expression plasmids and established stable cell lines, and the cells were further used for LC3 punctate measurement. Images were taken using a Nikon $\mathrm{Ti}$ inverted fluorescence microscope.

Reverse Transcription Polymerase Chain Reaction and Real-Time Polymerase Chain Reaction. The reverse transcription polymerase chain reaction assay was performed as in previous studies (Pi et al., 2010). The primers used for reverse transcription polymerase chain reaction and real-time polymerase chain reaction are listed in Supplemental Table 1.

Western Blot. Western blots were performed as previously described (Ye et al., 2017). Briefly, after 48-hour culture, quiescence was achieved in subconfluent cultures by removing the medium and washing with Hanks' balanced salt solution to remove residual serum, followed by incubation for an additional 4 hours in serum-free medium (Dulbecco's modified Eagle's medium/F-12 medium containing 0.1\% bovine serum albumin and about $1 \mathrm{mM}$ calcium; Thermo Fisher Scientific). After agonist treatment at the specified concentrations and duration, cells were washed twice with ice-cold PBS and scraped into $250 \mu \mathrm{l}$ of LDS sample buffer (Thermo Fisher Scientific) with $0.02 \mathrm{ml}$ per tablet of protease inhibitor (Thermo Fisher Scientific). Equal amounts of lysates were subjected to $10 \%$ SDS-PAGE, and target protein levels were determined by immunoblotting using antibodies. The antibodies used for these analyses are listed in Supplemental Table 2. Western blot intensity was analyzed by ImageJ software (https://imagej.nih.gov/ij/).

Confocal Microscopy Procedures. Cells stably expressing myctagged mouse WT GPRC6A (mGPRC6A $\mathrm{A}^{\mathrm{ICL} 3}$ _KGRKLP) or myc-tagged mouse GPRC6A ${ }^{\text {ICL3_KGKY }}$ were cultured in Dulbecco's modified Eagle's medium $+10 \%$ FBS supplemented with $200 \mu \mathrm{g} / \mathrm{ml}$ of G418. Cells plated on laminin-coated glass coverslips were preexposed to buffer or to $30 \mu \mathrm{M}$ Dyngo-4a for 30 minutes and then to $1 \mu \mathrm{M}$ testosterone for an additional 30 minutes ( $\mathrm{Li}$ et al., 2013). Thereafter, slides were fixed in PBS containing $4 \%$ paraformaldehyde and $4 \%$ sucrose $(\mathrm{pH}$ 7.4) for 10 minutes at room temperature (Bahouth and Nooh, 2017). To permeabilize the cells, fixed slides were incubated in HEPES-buffered saline solution containing $0.2 \%$ Triton $\mathrm{X}-100$ for 10 minutes at $4{ }^{\circ} \mathrm{C}$, followed by three washes in ice-cold PBS (Li et al., 2013). At this stage, coded slides were blocked with $5 \%$ bovine serum albumin in PBS for 30 minutes at room temperature. Cells expressing mGPRC6A ${ }^{\text {ICL3_KGRKLP }}$ or mGPRC6 $\mathrm{A}^{\text {ICL3_KGKY }}$ were incubated with 1:250 dilution of monoclonal anti-9E10 c-Myc antibody in PBS or 5\% FBS in PBS for permeabilized cells for 1 hour at room temperature. Similarly, cells expressing human GPRC6A (hGPRC6A ${ }^{\text {ICL3_KGKY }}$ ) were incubated with 1:250 dilution of rabbit anti-GPRC6A antibody in PBS for nonpermeabilized cells and 5\% FBS in PBS for permeabilized cells. Cells expressing mGPRC6A $\mathrm{A}^{\text {ICL3_KGRKLP }}$ or mGPRC6A $\mathrm{A}^{\text {ICL3_KGKY }}$ were incubated with 1:250 dilution of goat anti-mouse IgG conjugated to Alexa Fluor 555, while cells expressing hGPR6CA were incubated in 1:500 dilution of CF-633 conjugated to Donkey anti-rabbit IgG for 1 hour at room temperature. These antibodies were diluted in PBS for nonpermeabilized cells or PBS containing 5\% FBS for permeabilized cells. The slides were washed three times in PBS and then mounted on microscopic glass slides for confocal microscopy. Confocal fluorescence microscopy was performed at room temperature on coded slides and optical section thicknesses of $1.0 \mathrm{~mm}$ images were acquired by an Olympus FV1000 confocal microscope equipped with a 40 or $60 \AA$ oil immersion (numerical aperture 1.30) objective lens, using FV10-ASW 3.1 acquisition software (Olympus, Center Valley, PA) (Bahouth and Nooh, 2017). TIFF files of each image were exported and analyzed for pixel intensity and distribution by the ImageJ software. Each cell was partitioned by a line, every point of which was at a distance of $300 \mathrm{~nm}$ from the outer periphery of the cell, using the ImageJ software. This line formed the outer limit of the area used to index pixel intensity of internal GPRC6A in a given 
cell, while the area formed between the peripheral cell membrane and this line was used to index the distribution of pixels associated with cell surface GPRC6A (Gardner et al., 2007; Li et al., 2013; Bahouth and Nooh, 2017).

Statistics. Data were derived from image analysis that determined specific total pixels and pixels outside versus inside the $300-\mathrm{nm}$ partition that was drawn around the inner circumference of cardiomyocytes. The ratio of pixels residing outside the $300-\mathrm{nm}$ partition to that of the percentage of total pixels was calculated for each image. The average \pm S.E. of percentile pixel ratios from three separate experiments derived from 10 images/experiment ( $n=30$ images) is presented. Statistical comparison between two groups was performed by unpaired $t$ tests and for multiple groups by ANOVA (Tsvetanova and von Zastrow, 2014), followed by Bonferroni's test with a single pooled variance test in which the family-wise error rate was set at 0.05, using GraphPad Prism 7 software (GraphPad Software Inc.). Regarding the data obtained from western blot, cell proliferation, and autophagy assay, the statistical significance of differences between the two groups was calculated by using Student's $t$ test. Other statistical analyses performed were Dunnett's or Tukey-Kramer's tests, as post-hoc tests following ANOVA.

\section{Results}

Dose and Time Dependence of Testosterone-Mediated Activation of ERK/Phosphoinositide 3-Kinase/Protein Kinase B/mTORC1 Signaling in PC-3 Cells that Express the Endogenous GPRC6A ${ }^{\text {ICL3-KGKY }}$ Polymorphism. PC-3

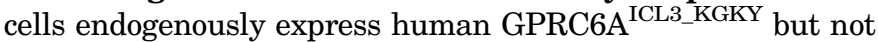
androgen receptor (AR) transcripts (Ye et al., 2017), making them a model to study the nongenomic, AR-independent effects of testosterone (Fig. 1A). The human prostate cancer cell, 22RV1, highly expressed GPRC6A and AR (Pi and Quarles,
A

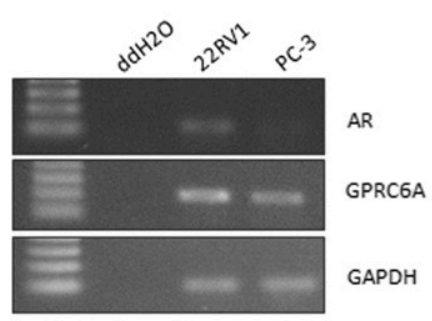

D

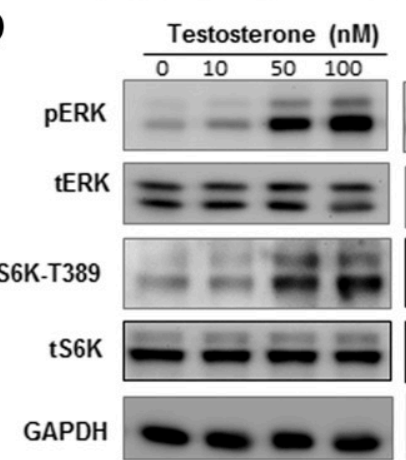

WT

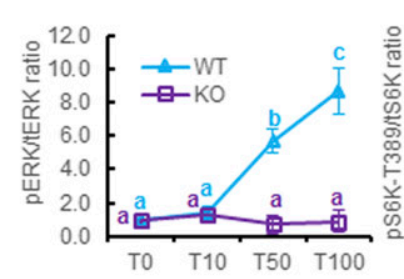

B

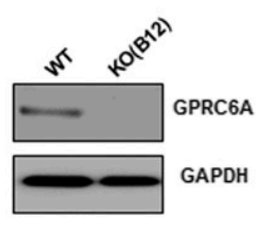

$\mathbf{F}$
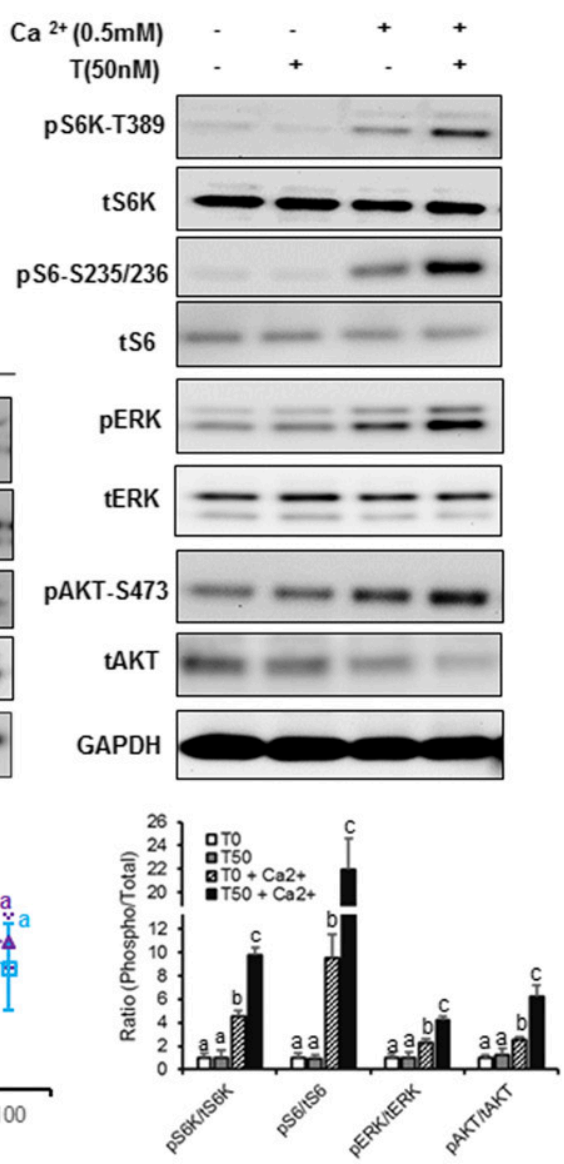

Fig. 1. GPRC6A directly mediated in testosterone-induced mTORC1 activation. (A) Reverse transcription polymerase chain reaction (PCR) analysis of $\mathrm{AR}$ and GPRC6A expression in PC-3 cells. 22Rv1 was employed as a positive control for the AR and GPRC6A expression human prostate cancer cell line. (B) Establishment of GPRC6A KO (B12) cell line by the CRISPR/Cas9 system. Western blot analysis of GPRC6A protein level in WT PC-3 cells (with Cas9 expression but no short guide RNA insert) and GPRC6A KO (B12) cells. (C) Real-time PCR of GPRC6A expression in WT PC-3 or KO PC-3 cells. Data are presented as mean \pm S.D. Each independent experiment was performed and replicated six times $(n=3)$. Different letters in the superscripts above the data points indicate significant differences between groups. Values sharing the same superscript letters are not significantly different from each other, and values with different superscript letters indicate significant differences between groups $(P<0.05$, Student's $t$ test.) (D) Knockout of GPRC6A abolished testosterone-induced mTORC1 activation. PC-3 WT cells and GPRC6A KO (B12) cells were treated with different concentrations of testosterone. Cells were incubated in Hanks' balanced salt solution (HBSS) buffer for 2 hours before 20-minute testosterone stimulation. Data are presented as mean \pm S.D. Each independent experiment was performed in biologic triplicates $(N=3)$. Statistical differences between groups are indicated by superscript letters $(P<0.05$, two-way ANOVA with Tukey's multiple comparisons test), as described for (C). (E) PC-3 cells were incubated in HBSS buffer for 2 hours before 20-minute treatment with dihydrotestosterone (DHT) at different concentrations. No activation is seen by DHT treatment. Statistical differences between groups are indicated by superscript letters, as described in (D). (F) $\mathrm{Ca}^{2+}$ is essential for the activation of mTORC1 and ERK and Akt phosphorylation. PC-3 cells were incubated in HBSS buffer with or without $0.5 \mathrm{mM} \mathrm{Ca}{ }^{2+}$ for 2 hours before 20 -minute testosterone stimulation. PC-3 cells were stimulated with HBSS buffer in the presence or absence of $0.5 \mathrm{mM} \mathrm{Ca}^{2+}$. Statistical differences between groups are indicated by superscript letters, as described in (D). 
2012 ); therefore, we used $22 \mathrm{RV} 1$ cells as the positive control. To create a PC-3 cell line with ablated GPRC6A, we used the CRISPR/Cas9 system to delete the hGPRC6A gene (Supplemental Figs. 1 and 2). We selected a PC-3 cell clone, termed B12 (PC-3/GPRC6A ${ }^{\mathrm{KO}-\mathrm{B} 12}$, which lacked the mRNA and protein of hGPRC6A (Fig. 1, B and C) and used it along with WT PC-3 cells to determine if ablation of hGPRC6A was associated with loss of downstream signaling by testosterone.

We found that the testosterone dose dependently activated ERK and p70S6 kinase (S6K) phosphorylation in WT human PC-3 cells expressing GPRC6A ${ }^{\text {ICL3_KGKY }}$ (Fig. 1D, left panel), and this response was lost in PC-3/GPRC6 $\mathrm{A}^{\mathrm{KO}-\mathrm{B} 12}$ cells (Fig. 1D, right panel), indicating that human GPRC6A was required for testosterone-mediated signaling responses in $\mathrm{PC}-3$ cells. Next, we tested the more specific AR ligand dihydrotestosterone that is derived from 5-alpha-reductase conversion of testosterone and in previous studies has been shown not to activate GPRC6A (Pi et al., 2010). Dihydrotestosterone failed to induce phosphorylation of ERK or S6K in PC-3 cells at concentrations ranging from 1 to $100 \mathrm{nM}$ (Fig. 1E), consistent with the notion that PC-3 cells lack the AR and in agreement with previous results showing that testosterone but not dihydrotestosterone activated downstream signaling by the hGPRC6A (Pi et al., 2015).

Because the VFT and seven-transmembrane structures of GPRC6A predict activation by cations and allosteric modulators, we reexamined if $\mathrm{Ca}^{2+}$ is required for testosterone-mediated activation of hGPRC6A (Wellendorph and Bräuner-Osborne, 2009; Pi et al., 2010; Oury et al., 2013; White et al., 2013). For these studies, PC-3 cells were incubated in a buffer with or without $0.5 \mathrm{mM} \mathrm{Ca}^{2+}$ and then exposed to testosterone. We found that testosterone failed to induce phosphorylation of ERK, protein kinase B (AKT), S6K, or ribosomal protein S6 (S6) signaling in the buffer without $\mathrm{Ca}^{2+}$ (Fig. 1F). $\mathrm{Ca}^{2+}$ alone weakly stimulated phosphorylation of these downstream signals, but the effects of testosterone on $\mathrm{Ca}^{2+}$ were additive, leading to robust induction of phosphorylation, as previously reported (Pi et al., 2010).

The time course of testosterone-mediated activation of endogenous hGPRC6A was rapid in onset. We observed increased phosphorylation of both ERK and AKT within 2 minutes with maximal activation by 10 minutes that was sustained throughout the 30-minute observation period (Fig. 2A).

ERK and AKT are upstream of mTORC1 (Mendoza et al., 2011), an evolutionarily conserved nutrient-sensing pathway (Efeyan et al., 2012; Ben-Sahra and Manning, 2017). Phosphorylations of S6K at Thr389, S6 at Ser235/236, and eukaryotic translation initiation factor $4 \mathrm{E}$ binding protein 1 (4E-BP1) at Ser65 are characteristic downstream markers for activation of the mTORC1 pathway (Mendoza et al., 2011). Testosterone at concentrations of $50 \mathrm{nM}$ time dependently increased the phosphorylation of pS6K-Thr389, pS6-Ser235/236, and p4E-BP1-Ser65, and their phosphorylation was activated as early as 2 minutes (Fig. $2 \mathrm{~B}$ ).

To further confirm that ERK and AKT mediated testosteroneinduced mTORC1 activation, PC-3 cells were treated with
A

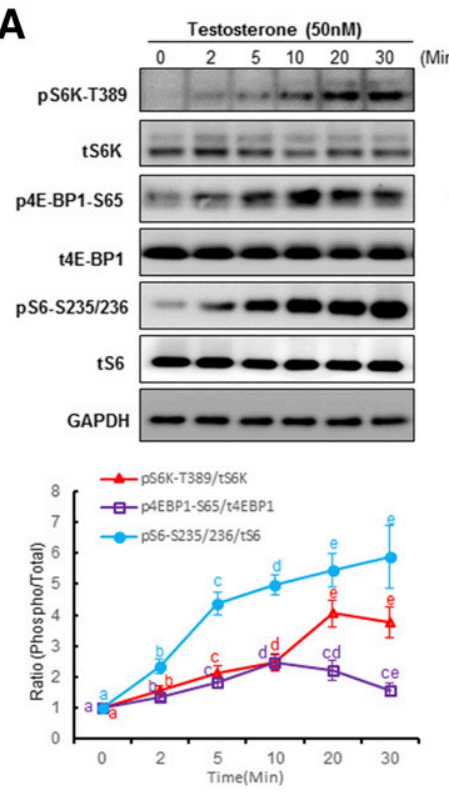

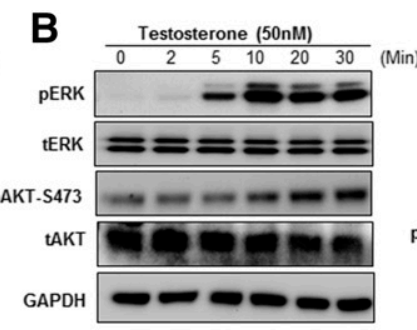

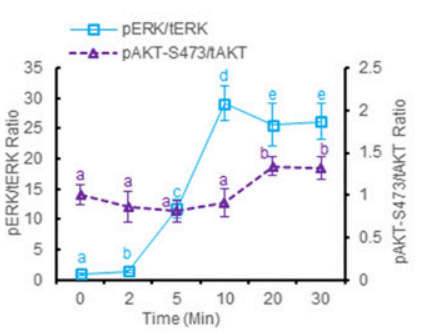

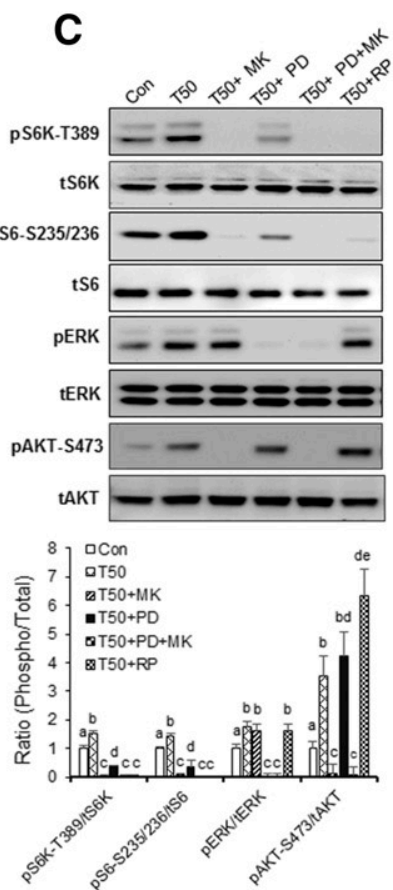
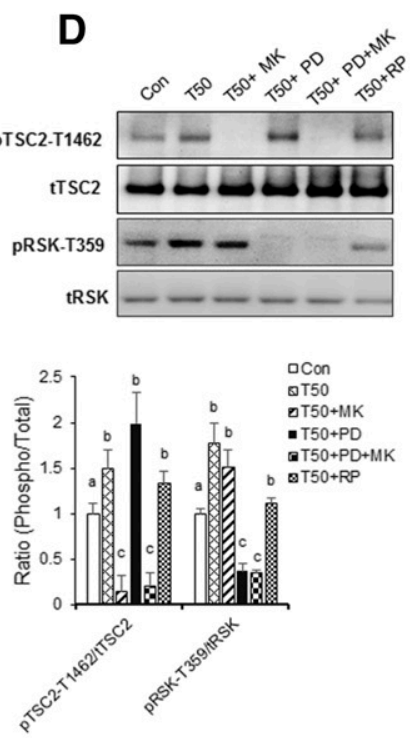

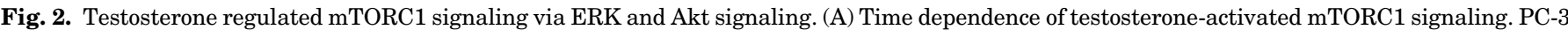

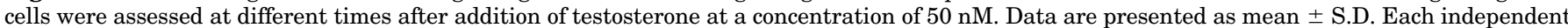

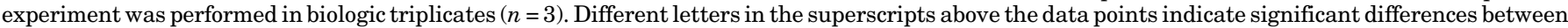

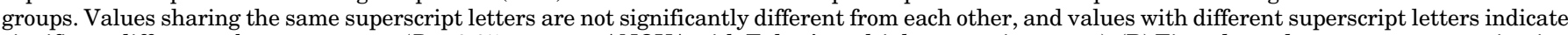

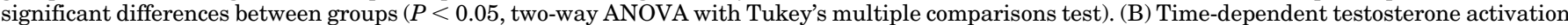

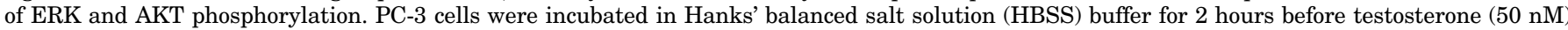

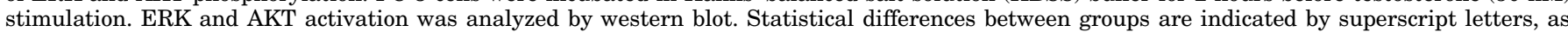

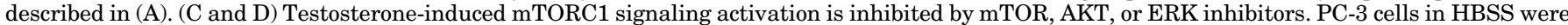

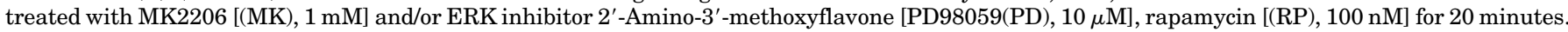

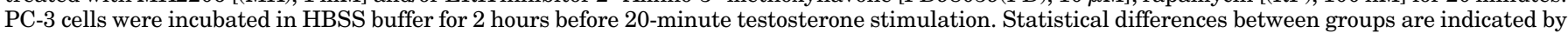
superscript letters, as described in (A). 
testosterone in the presence of 2 '-Amino-3'-methoxyflavone (PD98059,ERK inhibitor), and/or 8-[4-(1-aminocyclobutyl)phenyl]-9-phenyl-2H-[1,2,4] triazolo[3,4-f] [1,6]naphthyridin3-one [(MK2206), an AKT inhibitor]. Testosterone-induced mTORC1 activation was partially blocked by the ERK inhibitor (2'-Amino-3' -methoxyflavone,PD98059) and completely inhibited by the AKT inhibitor (MK2206) (Fig. 2C). Treatment with the potent AKT inhibitor MK2206 completely inhibited AKT-S473 phosphorylation, but not ERK phosphorylation. In addition, MK2206 inhibited the phosphorylation of S6 at pS6K-T389 and pS6-S235/236, which is a downstream target of mTORC1 activation (Fig. 2C). 2' Amino-3'-methoxyflavone (PD98059) completely blocked ERK phosphorylation, but had no effect on AKT phosphorylation, and partially inhibited S6 phosphorylation (Fig. 2C). Rapamycin, a mammalian target of rapamycin (mTOR) inhibitor, blocked testosterone-induced S6 phosphorylation in PC-3 cells without affecting ERK or AKT phosphorylation (Fig. 2, C and D).

To define pathways between ERK and AKT activation of mTORC1, we examined the effects of testosterone on phosphorylation of tuberous sclerosis complex 2 and mitogenactivated protein kinase-activated protein $90 \mathrm{kDa}$ ribosomal S6 kinase(RSK). Testosterone significantly stimulated ribosomal S6 kinase phosphorylation in PC-3 cells, and this response was completely blocked by the ERK inhibitor, partially inhibited by rapamycin, and not affected by the AKT inhibitor. Conversely, testosterone also induced tuberous sclerosis complex 2 phosphorylation at Thr1462, and this response was blocked by the AKT inhibitor but not the ERK inhibitor (Fig. 2D). These findings suggest that ERK/90 kDa ribosomal $\mathrm{S} 6$ kinase and $\mathrm{AKT}$ are two independent pathways involved in testosterone-induced mTORC1 activation in PC-3 cells.

GPRC6A Regulates Cell Proliferation and Autophagy through mTORC1 Signaling. Since mTORC1 is a master regulator of cell growth, metabolism, and survival (Laplante and Sabatini, 2009), we determined the role of mTORC1 in mediating the function of hGPRC6A in PC-3 cells.

First, we examined the effects of testosterone on cell survival and proliferation in the WT PC-3/GPRC6 $\mathrm{A}^{\text {ICL3-KGKY }}$ and GPRC6A KO PC-3/GPRC6 ${ }^{\mathrm{KO}-\mathrm{B} 12}$ cell lines. These cells were seeded at the same density and incubated for 2 days in the presence or absence of testosterone (Cvicek et al., 2016). We consistently counted fewer PC-3/GPRC6A ${ }^{\mathrm{KO}-\mathrm{B} 12}$ cells compared with WT PC-3 hGPRC6A cells leading to impaired cell proliferation and/or decreased survival. We speculate that these effects were mediated by hGPRC6A, because testosterone significantly increased the number of cells in WT PC-3 cells expressing the endogenous GPRC6 $\mathrm{A}^{\text {ICL3-KGKY }}$ variant but not in PC-3/GPRC6 $\mathrm{A}^{\mathrm{KO}-\mathrm{B} 12}$ cells (Fig. 3A). Testosterone also induced expression of the cell proliferation marker Ki-67 (Fig. 3B) and phosphorylation of 4E-BP1 and eIF4G, two effectors of mTORC1 signaling that are responsible for regulating the proliferation of PC-3/GPRC6 $\mathrm{A}^{\text {ICL3-KGKY }}$ cells. However, in hGPRC6A-null PC-3 (PC-3/GPRC6A ${ }^{\text {KO-B12 }}$ ) cells, the effect of testosterone on these parameters was completely abolished (Fig. 3B).

Next, we examined if human GPRC6A was involved in regulating autophagy in $\mathrm{PC}-3$ cells because mTORC1 is a major inhibitor of autophagy, and testosterone has been shown to inhibit autophagy (Tomaszewski et al., 2015). Since mTORC1 suppressed autophagy via phosphorylating Unc-51-like autophagy activating kinase-1 ULK1 at Ser757, we examined if testosterone would further increase the phosphorylation of ULK-1 on Ser757. Testosterone induced a time-dependent increase in the phosphorylation of ULK-Ser757, achieving a maximal response by 30 minutes (Fig. 3C). Testosteronemediated increase in the phosphorylation of ULK-Ser757 was observed in WT and PC-3/GPRC6 $\mathrm{A}^{\text {ICL3-KGKY }}$ cells, but not in GPRC6A null PC-3/GPRC6A ${ }^{\text {KO-B12 }}$ cells (Fig. 3D).

The autophagy-related protein LC3 is a widely used marker of autophagosomes, where the lapidated form of LC3-II indexes autophagic activity (Yoshii and Mizushima, 2017). Western blot analysis showed that phosphorylation of lipidated LC3-II was decreased in PC-3/GPRC6A ${ }^{\text {ICL3-KGKY cells }}$ after testosterone stimulation, whereas basal phosphorylation was increased in vehicle-treated PC-3/GPRC6A $\mathrm{A}^{\mathrm{KO}-\mathrm{B} 12}$ cells that exhibited no response to testosterone (Fig. 3D). PC-3/GPRC6A $\mathrm{A}^{\mathrm{KO}-\mathrm{B} 12}$ cells also exhibited increased formation of LC3 autophagosomes, while testosterone reduced LC3-II expression in PC-3/GPRC6 $\mathrm{A}^{\text {ICL3-KGKY }}$ cells, an effect blocked by deletion of GPRC6A blocked in PC-3/GPRC $6 \mathrm{~A}^{\mathrm{KO}-}$ B12 (Fig. 3E).

Collectively, these results unequivocally establish the function of endogenous human GPRC6 $\mathrm{A}^{\text {ICL3-KGKY }}$, and contrast with the reported lack of function of a transfected hGPRC6A construct, which was found to be retained intracellularly (Jørgensen et al., 2017). We propose that hGPRC6A is an example of a GPCR that exhibits ligand-dependent signaling after endocytosis (Thomsen et al., 2016; Cahill et al., 2017).

Cellular Localization and Ligand-Dependent Activation of the GPRC6A KGKY and RKLP Polymorphisms. To examine the impact of the KGKY and KGRKLP motifs on ligand-dependent endocytosis, we transfected WT human GPRC6A (GPRC6A ${ }^{\text {ICL3_KGKY }}$ ), Myc-tagged WT mouse

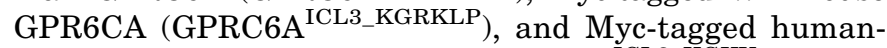
ized mouse GPRC6A (Myc-mGPRC6A ${ }^{\text {ICL3_KGKY), which }}$ were created by replacing KGRKLP with KGKY in the ICL3 of mGPRC6A into HEK-293 cells. The cells were fixed and then either used as such or permeabilized as described in Materials and Methods. The cells were then incubated with primary monoclonal (9E10) anti-Myc or rabbit antihuman GPR6CA antibodies, followed by secondary fluorescent anti-mouse or anti-rabbit IgG. Confocal fluorescence microscopy indicated that in HEK-293 cells, ancestral mGPRC6A (GPRC6A ${ }^{\text {ICL3_KGRKLP) }}$ ) pixels were predominately located $(70 \% \pm 14 \%)$ in the cell surface membrane of intact or permeabilized HEK-293 cells (Fig. 4A, images a and b, respectively). In contrast, in HEK-293 cells, humanized mouse GPRC6A (mGPRC6A ${ }^{\text {ICL3_KGKY }}$ ) (Fig. 4A, images c and d), and human GPRC6A (Fig. 4A, images e and f) were mostly intracellular. These receptors were distributed by $\sim 12 \%$ and $17 \%$ on the cell surface and by $88 \% \pm 17 \%$ and $83 \% \pm 15 \%$ internally (Fig. 4B), respectively ( $n=30$ images derived from three separate experiments).

Consistent with findings in classic GPCR, activation of mGPRC6A with testosterone induced the redistribution of the pixels from the membrane to internal puncta that had a diameter of 300-500 $\mathrm{nm}$, commonly associated with endosomal structures (Fig. 5A, images a and b). In contrast, addition of testosterone, which activates hGPRC6A, did not alter the distribution of internal humanized mGPRC6A $\left(\right.$ GPRC6A ${ }^{\text {ICL3_KGKY }}$ (Supplemental Fig. 3). 
Translocation of mGPRC6A in response to testosterone was inhibited in cells pretreated with the potent dynamin inhibitor Dyngo-4a (Robertson et al., 2014) (Fig. 5A, image c). To confirm Dyngo-4a blocks mGPRC6Amediated testosterone-induced translocation and function, we compared the functions of mGPRC6A and hGPRC6A in testosterone-induced intracellular ERK phosphorylation. In contrast, we found that Dyngo-4a enhanced testosteroneinduced ERK phosphorylation in mGPRC6A, but inhibited testosterone-induced ERK activity in hGPRC6A-transfected HEK-293 cells (Fig. 5B).

$\beta$-Arrestins are multifunctional endocytic adaptors and signal transducers (Lefkowitz and Shenoy, 2005). Next, we studied if $\beta$-arrestins are involved in GPRC6A endocytosis using the $\beta$-arrestin inhibitor, Barbadin (Beautrait et al., 2017). Like Dyngo-4a, Barbadin (10 $\mu \mathrm{M})$ enhanced testosterone-induced ERK phosphorylation in mGPRC6Atransfected HEK-293 cells, but inhibited testosteroneinduced ERK phosphorylation in hGPRC6A-transfected HEK-293 cells (Fig. 5C, upper panel). Interestingly, Barbadin also increased activation of $\mathrm{S} 6 \mathrm{~K}$ in $\mathrm{mGPRC} 6 \mathrm{~A}$, but decreased S6K phosphorylation in hGPRC6A-transfected HEK-293 cells (Fig. 5C, middle panel).
These findings are consistent with prior studies showing desensitization of mouse GPRC6A signaling through a $\beta$-arrestin-dependent classic rapid recycling mechanism, and the findings of Jørgensen et al. (2017) showing that the $\mathrm{KY}$ insertion/deletion in GPRC6A is mostly retained intracellularly and recycles through a $\beta$-arrestin-dependent slow recycling mechanism (Lefkowitz and Shenoy, 2005; Pi et al., 2005).

Finally, we compared the effect of KGKY on western blot migration of epitope-tagged mGPRC6A, humanized mouse GPRC6A, and human GPRC6A using Myc antibody. The estimated molecular weight of the GPRC6A monomer is $110 \mathrm{kDa}$, and GPRC6A is known to form a dimer with molecular weight of $\sim 220 \mathrm{kDa}$ (Nørskov-Lauritsen et al., 2015; Ye et al., 2017). We found that the human GPRC6A dimerized to a lesser extent compared with the mouse GPRC6A, as evidenced by the altered distribution of GPRC6A monomers and dimers in HEK-293 cells transfected with these constructs (Fig. 5D). Interestingly, both WT mouse mGPRC6A $\mathrm{A}^{\text {ICL3-KGRKLP }}$ and humanized mGPRC6A ${ }^{\text {ICL3-KGKY }}$ showed equal amounts of 110 and $220 \mathrm{kDa}$ protein bands, corresponding to monomer and dimer migration (1.23 or 1.18 , dimer/monomer ratio,
A

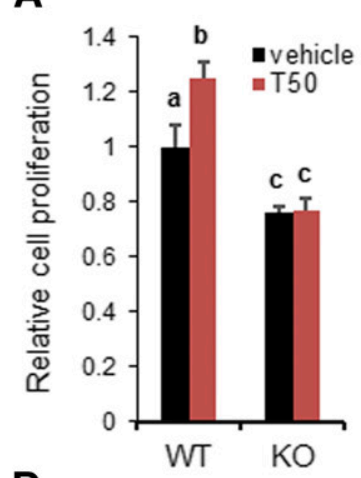

D

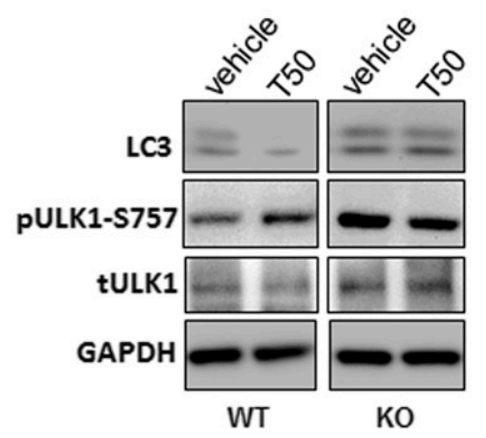

B

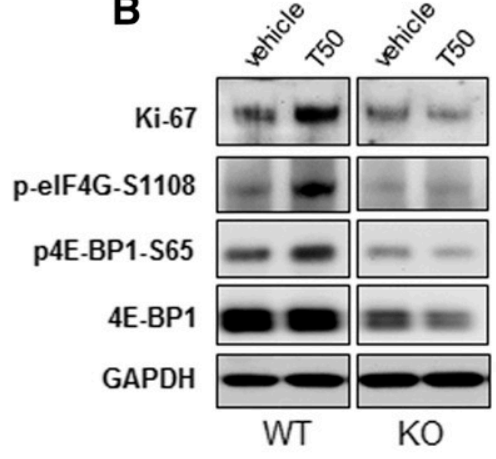

E

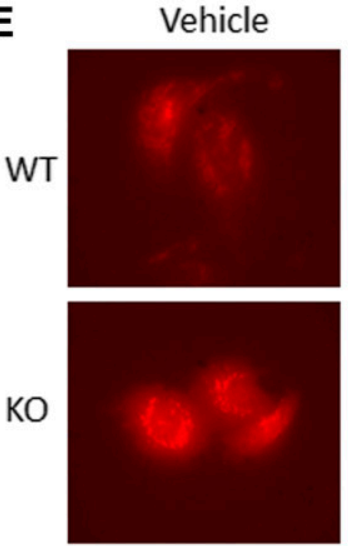

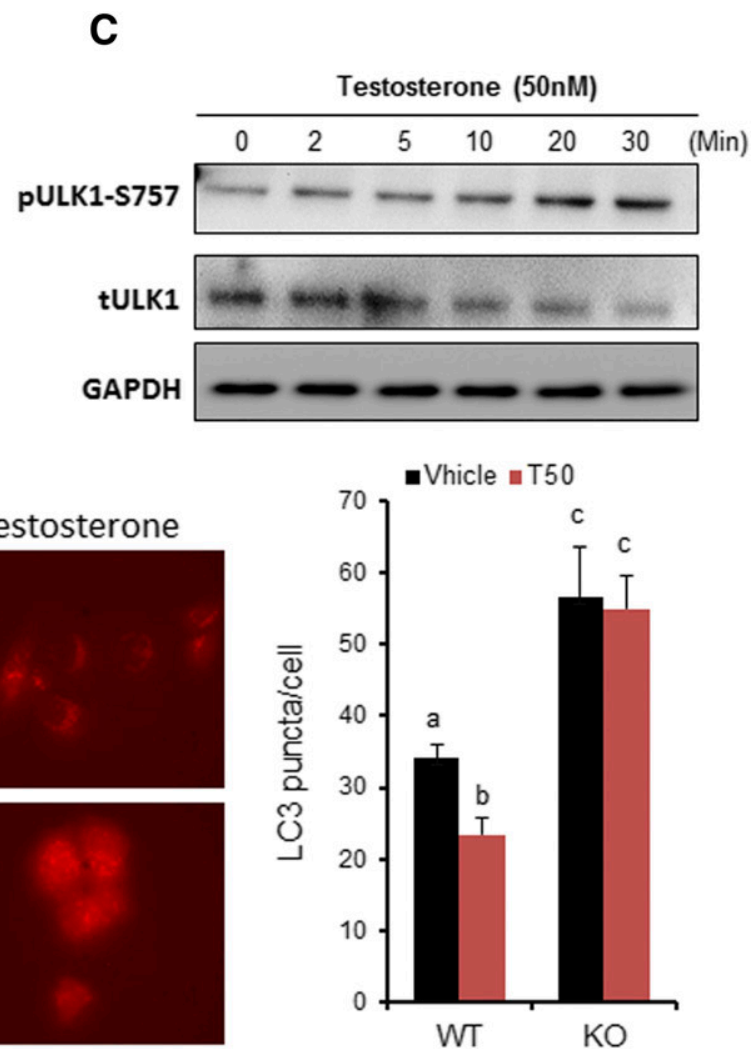

Fig. 3. GPRC6A mediated in testosterone-induced cell proliferation and autophagy. (A) Cell proliferation of PC-3 WT cells and KO (B12) cells using the 3-(4,5-dimethylthiazol-2-yl)-2,5-diphenyltetrazolium bromide (MTT) method. On day 0, $3 \times 10^{3}$ per well of cells were seeded in 96-well plates. Cell viability was measured on day 2. Cells were cultured in complete RPMI 1640 media with or without $50 \mathrm{nM}$ of testosterone (T50). Data are mean \pm S.D. $(n=8)$. Different letters in superscripts above the data points indicate significant differences between groups. Values sharing the same superscript letters are not significantly different from each other, and values with different superscript letters indicate significant differences between groups $(P<0.05$, two-way ANOVA with Tukey's multiple comparisons test). (B) Western analysis of cell proliferation marker Ki67 expression and mTORC1 activation. Cells were cultured in complete RPMI 1640 media with or without T50 for 48 hours. (C) Time course of testosterone on Unc-51 like autophagy activating kinase 1 (ULK1) activation. Phosphorylation of ULK1 at Ser757 was measured using western blot. PC-3 WT cells were incubated in Hanks' balanced salt solution (HBSS) buffer for 2 hours before 20-minute T50 stimulation. (D) Western blot analysis of LC3B level and ULK1 activation. Cell autophagy was induced by 5-hour starvation in HBSS with $50 \mu \mathrm{M}$ chloroquine (Lee et al., 2007). (E) Representative LC3 punctate images of PC-3 WT cells and PC-3 GPRC6A-KO (B12) cells stably expressing LC3-RFP. Autophagy is induced by 5-hour starvation in HBSS buffer with $50 \mu \mathrm{M}$ chloroquine (Lee et al., 2007). Scale bar, $20 \mu \mathrm{m}$. LC3 punctate was quantitated by ImageJ software. Data are mean \pm S.D. of LC3 punctate/cell. Statistical differences between groups are indicated by superscript letters, as described in (A). 
respectively), whereas WT human GPRC6A ${ }^{\text {ICL3-KGKY }}$ showed a greater ratio of monomer to dimer (0.32 dimer/monomer ratio), suggesting that the polymorphisms in the ICL3 are not controlling dimerization (Fig. 5D).

\section{Discussion}

A newly evolved human GPRC6A ortholog with the ICL3_KGKY polymorphism is reported to be retained intracellularly and to lack ligand-dependent activation (Jørgensen et al., 2017). These negative findings, along with failure to identify any association between GPRC6A mutations and human diseases in a Danish cohort have raised questions about the biologic relevance of GPRC6A in humans. However, our data show that the human GPRC6A ${ }^{\text {ICL3_KGKY }}$ variant, although predominantly intracellular, can be detected in the plasma membrane, and more importantly is functional as evidenced by its activation by testosterone and other agonists, as previously reported ( $\mathrm{Pi}$ et al., 2016a, 2018a,b).

The current studies extend our understanding of the important difference between GPRC6A membrane locations imparted by replacement of the ancestral RKLP sequence with the KGKY insertion/deletion in the ICL3. We show that mouse GPRC6A with the ancestral RKLP polymorphism in the ICL3 acts like a classic GPCR in that mGPRC6A is predominately expressed in the cell surface membrane and undergoes ligand-dependent internalization, perhaps via clathrin-coated pit-mediated endocytosis. On the other hand, human GPRC6A ${ }^{\text {ICL3_KGKY }}$ is present at low levels in the cell membrane $(\sim 20 \%)$ and is predominately located $(\sim 80 \%)$ in the endosome-like intracellular punctuate structures, consistent with the observation that human GPRC6A is largely retained intracellularly and undergoes slow recycling from the endosome (Jacobsen et al., 2017). The molecular mechanism whereby replacing the KGRKLP sequence with KGKY leads to intracellular retention needs further study. Since the KGRKLP motif is present in family $\mathrm{C}$ members that undergo desensitization and recycling to the plasma membrane, it is tempting to speculate that the KGRKLP sequence represents a membrane retention motif, and the replacement with the KGKY motif leads to loss of binding to essential proteins, leading to GPCR cycling to endosomes (Lefkowitz and Shenoy, 2005).

Regardless, and most importantly, in contrast to the purported lack of ligand-dependent activation of a transfected human GPRC6A (Jørgensen et al., 2017), we found that endogenous human GPRC6A in PC-3 cells responds to the agonist testosterone by activating ERK and phosphoinositide 3-kinase/AKT signaling pathways. More importantly, ligand activation of GPRC6A is linked to downstream mTOR activation. The cell surface expressed human GPRC6A may likely account for our previous observations showing that coexpression of $\beta$-arrestin 1 and 2, C3 toxin, or pertussis toxin pretreatment blocked the effects of cations to activate transfected human GPRC6A (Pi et al., 2005). The endosomal signaling by internalized GPRC6A is similar to the evolutionarily conserved pathway in which L-arginine activates mTORC1 signaling in lysosomes from yeast to mammals (Kapahi et al., 2010; Efeyan et al., 2012; Saxton and Sabatini, 2017). Similar to the lysosomal nutrient sensing pathway, testosterone activation of GPRC6A in the endosomes may also result in the inhibition of autophagy and induction of anabolic cellular responses through activation of S6 kinase and inhibition of repressor polypeptide 4E-BP1 and ULK1.

Our current findings are consistent with prior observations that humanizing the mouse GPRC6A by replacing the
A
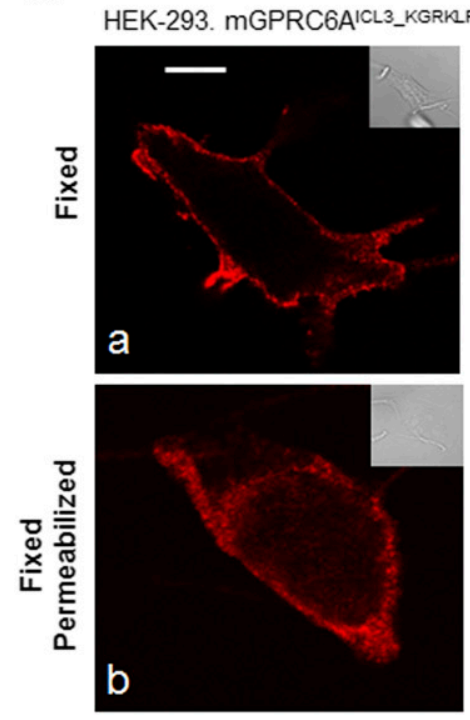
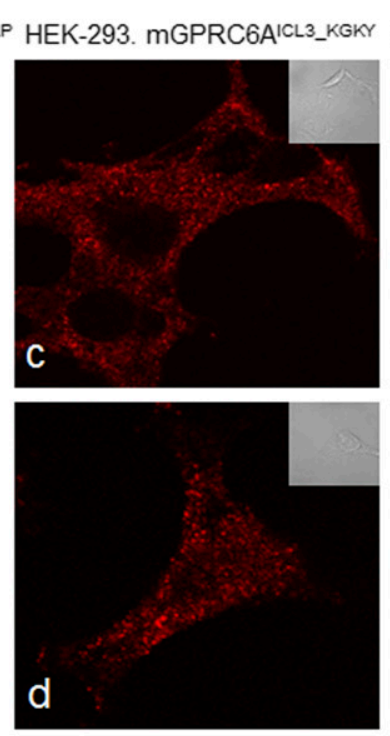
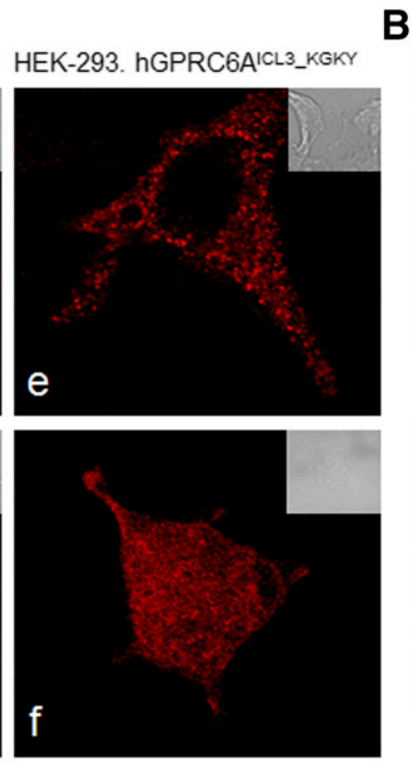

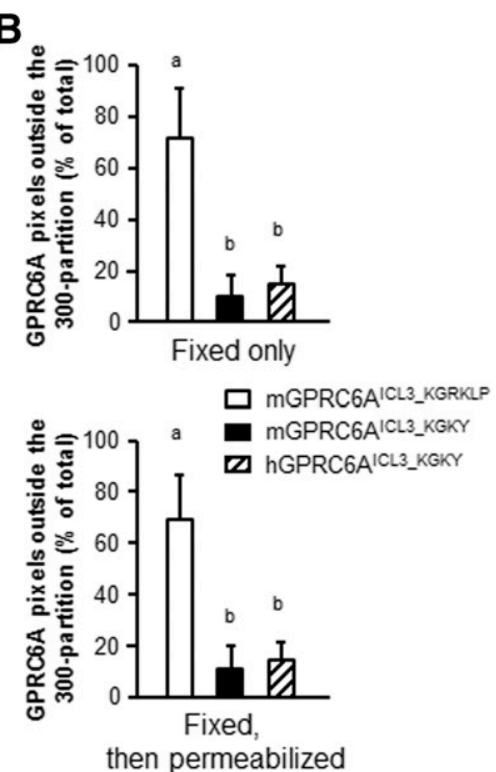

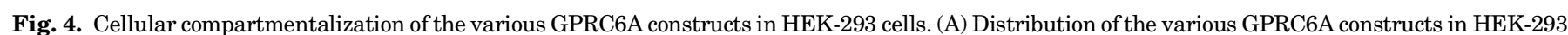

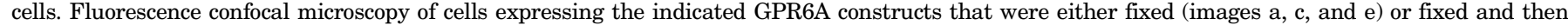

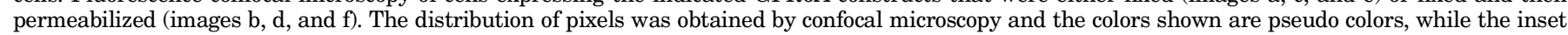

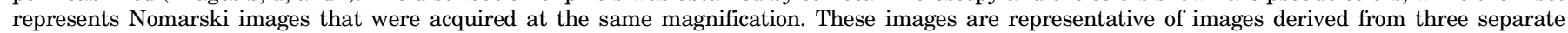

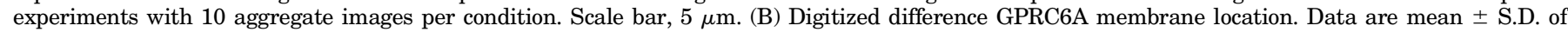

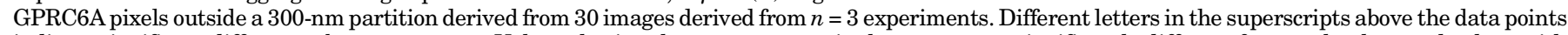

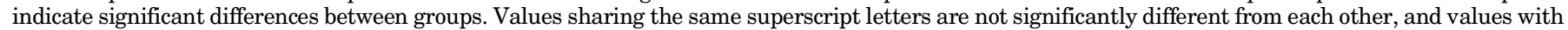
different superscript letters indicate significant differences between groups $(P<0.05$, two-way ANOVA with Tukey's multiple comparisons test). 
KGRKLP sequence with KGKY prolonged mTOR signaling, suggesting the internalized KGKY polymorphism is a gain-offunction variant (Ye et al., 2017), but not consistent with the reported agonist-independent internalization and slow recycling from the endosome of human GPRC6A reported by others (Jacobsen et al., 2017).

The structural basis for sensing of cations and L-arginine resides in the VFT, and testosterone, Ocn, and other ligands are imparted by the VFT and seven-transmembrane domain structure (Pi et al., 2005, 2015, 2016a, 2018a). The inability to show ligand-dependent activation of GPRC6A by other investigators may be due to inclusion of a wash step that depleted essential cofactors necessary for ligand activation of GPRC6A. Consistent with this possibility, we found that testosterone activation of GPRC6A is dependent on media calcium concentration of at least $0.5 \mathrm{mM}$. Physiologically, GPRC6A is always in the presence of calcium above the activating threshold, whereas the concentrations of testosterone, Ocn, and other ligands may vary to activate GPRC6A. Our studies examine the function of GPRC6A in PC-3 cells that do not express the AR; however, we have shown that GPRC6A has a similar function in 22RV1 cells that express the AR (Pi and Quarles, 2012).
Thus, rather than being a nonfunctional variant, human GPRC6A appears to belong to the growing examples of GPCRs that exhibit endosomal signaling after ligand-mediated internalization (Thomsen et al., 2016; Cahill et al., 2017). In the case of GPRC6A ${ }^{\text {ICL3_KGKY }}$ the internal signaling is linked to ERK and mTOR, suggesting that the KGKY polymorphism may have evolved to provide a means for a GPCR to activate mTORC1 in endosomes/lysosomes. Additional studies are needed to define the intracellular compartments in which GPRC6A activates mTOR signaling.

Regardless, the potential biologic significance of GPRC6A is shown by studies of mouse GPRC6A, indicating important roles in regulating energy metabolism and prostate cancer progression (Pi et al., 2010, 2011, 2016b; Pi and Quarles, 2012; Oury et al., 2013; Di Nisio et al., 2017). GPRC6A antagonists would inhibit both AKT/mTOR and ERK/mitogen-activated protein kinase signaling, which is an effective method for treating hormone-refractory prostate cancer (Kinkade et al., 2008). If the human GPRC6A ${ }^{\text {ICL3_KGKY }}$ has similar functions to mouse GPRC6A, it may account for racial disparities in susceptibility to metabolic disorders and prostate cancer (Cho, 2011; Laplante and Sabatini, 2012).
A
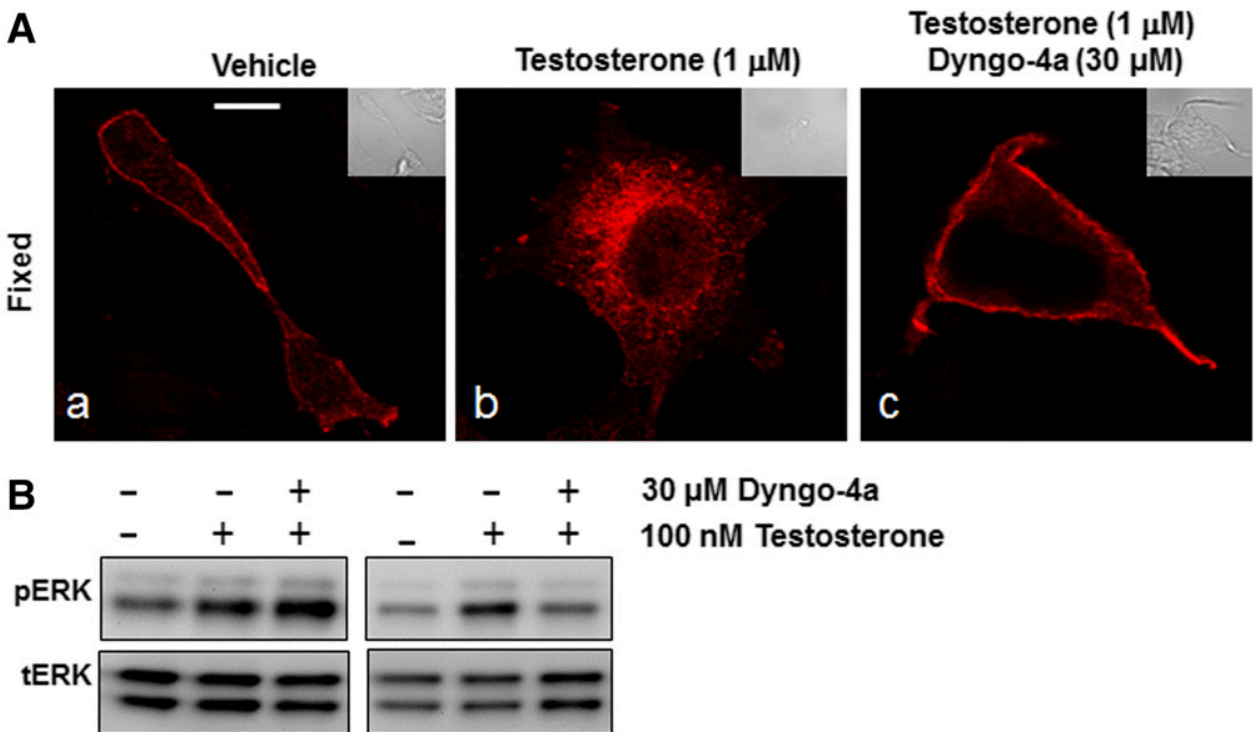

$30 \mu \mathrm{M}$ Dyngo-4a

$100 \mathrm{nM}$ Testosterone

HEK-293

HEK-293.

mGPRC6A'ICL3_KGRKLP hGPRC6A'ICL3_KGKY

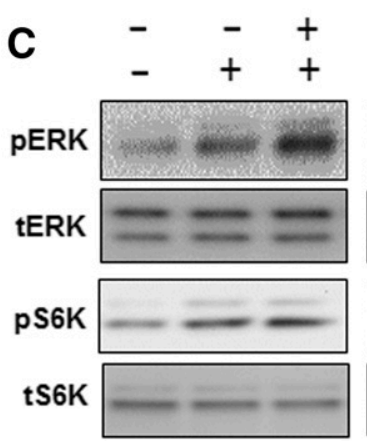

HEK-293.

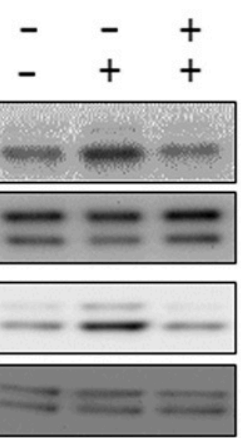

$10 \mu \mathrm{M}$ Barbadin

$100 \mathrm{nM}$ Testosterone

mGPRC6A ${ }^{\text {ICL3_KGRKLP }}$
Fig. 5. Comparison of intracellular function of mouse and human GPRC6A in HEK-293 cells. (A) Dyngo-4a inhibited GPRC6A ligand; testosterone induced receptor internalization. The slides were pretreated with diluent or $30 \mu \mathrm{M}$ Dyngo4a for 30 minutes and then exposed to $1 \mu \mathrm{M}$ testosterone for 30 minutes and fixed (images b and c, respectively). Scale bar, $5 \mu \mathrm{m}$. (B and C) Comparison of Dyngo4a and Barbadin regulated GPRC6A-mediated ligand; testosterone induced ERK and S6K phosphorylation in HEK-293 cells stably transfected with WT mouse and human GPRC6A (mGPRC6A ${ }^{\text {ICL3_KGRKLP }}$ and hGPRC6. ${ }^{\text {ICL3_KGKY }}$ cDNAs. Cells were preincubated with vehicle, Dyngo-4a (30 $\mu \mathrm{M}$ (Ab120689; Abcam), or Barbadin $(10 \mu \mathrm{M})$ (Axon2774; Axon Medchem) for 20 minutes after testosterone treatment. (D) Comparison of dimerization of the various GPRC6As in HEK-293 cells, WT mouse GPRC6A (mGPRC6A ${ }^{\text {ICL3_KGRKLP) }}$ vs. humanized mouse GPRC6A (mGPRC6A ${ }^{\text {ICL3_KGRKLP) }}$ or human GPRC6A (hGPRC6A ${ }^{\text {ICL3_KGKY), }}$ analyzed by western blots: $16 \mu \mathrm{g}$ of each plasmid cDNA was transfected into HEK293 cells $\left(1.5 \times 10^{6}\right.$ cells $/ 100 \mathrm{~mm}$ dish $)$ and $100 \mu \mathrm{g}$ of each total protein sample was loaded on $3 \%-8 \%$ SDS-PAGE gels. AntiMyc antibody was used in the western blot analysis. These images are representative of images derived from three separate experiments. 
Presently, clinical studies show that levels of the GPRC6A ligand, Ocn, are inversely associated with glycemic status, insulin resistance, and obesity in humans (Pittas et al., 2009; Foresta et al., 2011; Iki et al., 2012). Genome-wide association studies find association with GPRC6A polymorphisms with insulin resistance (Di Nisio et al., 2017) testicular failure (Oury et al., 2013; De Toni et al., 2016), and prostate cancer (Takata et al., 2010; Long et al., 2012). However, a recent study of a Danish cohort failed to identify any association between GPRC6A inactivating mutations and human disease (Jørgensen et al., 2017). Analysis of genome-wide association studies is confounded because the KGKY polymorphism is the referent sequence in humans and the RKLP polymorphism is not detected by current gene chips used to genotype patient cohorts.

In conclusion, the emergence of signaling of endogenous hu GPRC6A $\mathrm{A}^{\mathrm{ICL} 3 \_K G K Y}$ only in hominids and its preservation during evolution indicates selection pressures that modified the functions of GPRC6A to promote ligand-depedent intracellular signaling and coupling to ERK and mTOR. Further studies are needed to establish the clinical relevance of GPRC6A in human pathophysiology.

\section{Acknowledgments}

We acknowledge Dr. Malia B. Potts for gifting the GFP-RFP-LC-3 plasmid.

\section{Authorship Contribution}

Participated in research design: $\mathrm{Ye}, \mathrm{Pi}$, Quarles.

Conducted experiments: $\mathrm{Ye}, \mathrm{Pi}$, Nooh, Bahout.

Performed data analysis: $\mathrm{Ye}, \mathrm{Pi}$, Bahout, Quarles.

Wrote or contributed to the writing of the manuscript: $\mathrm{Ye}, \mathrm{Pi}$, Bahout, Quarles.

\section{References}

Bahouth SW and Nooh MM (2017) Barcoding of GPCR trafficking and signaling through the various trafficking roadmaps by compartmentalized signaling networks. Cell Signal 36:42-55.

Beautrait A, Paradis JS, Zimmerman B, Giubilaro J, Nikolajev L, Armando S, Kobayashi H, Yamani L, Namkung Y, Heydenreich FM, et al. (2017) A new inhibitor of the $\beta$-arrestin/AP2 endocytic complex reveals interplay between GPCR internalization and signalling. Nat Commun 8:15054.

Ben-Sahra I and Manning BD (2017) mTORC1 signaling and the metabolic control of cell growth. Curr Opin Cell Biol 45:72-82.

Cahill TJ III, Thomsen AR, Tarrasch JT, Plouffe B, Nguyen AH, Yang F, Huang LY, Kahsai AW, Bassoni DL, Gavino BJ, et al. (2017) Distinct conformations of GPCR$\beta$-arrestin complexes mediate desensitization, signaling, and endocytosis. Proc Natl Acad Sci U S A 114:2562-2567.

Cho $\mathrm{CH}$ (2011) Frontier of epilepsy research-mTOR signaling pathway. Exp Mol Med 43:231-274.

Cvicek V, Goddard WA III, and Abrol R (2016) Structure-based sequence alignment of the transmembrane domains of all human GPCRs: phylogenetic, structural and functional implications. PLoS Comput Biol 12:e1004805.

De Toni L, De Filippis V, Tescari S, Ferigo M, Ferlin A, Scattolini V, Avogaro A, Vettor R, and Foresta C (2014) Uncarboxylated osteocalcin stimulates 25-hydroxy vitamin D production in Leydig cell line through a GPRC6a-dependent pathway. Endocrinology 155:4266-4274.

De Toni L, Di Nisio A, Speltra E, Rocca MS, Ghezzi M, Zuccarello D, Turiaco N, Ferlin A, and Foresta C (2016) Polymorphism rs2274911 of GPRC6A as a novel risk factor for testis failure. J Clin Endocrinol Metab 101:953-961.

Di Nisio A, Rocca MS, Fadini GP, De Toni L, Marcuzzo G, Marescotti MC, Sanna M, Plebani M, Vettor R, Avogaro A, et al. (2017) The rs2274911 polymorphism in GPRC6A gene is associated with insulin resistance in normal weight and obese subjects. Clin Endocrinol (Oxf) 86:185-191.

Efeyan A, Zoncu R, and Sabatini DM (2012) Amino acids and mTORC1: from lysosomes to disease. Trends Mol Med 18:524-533.

Ferron M, Wei J, Yoshizawa T, Del Fattore A, DePinho RA, Teti A, Ducy P, and Karsenty G (2010) Insulin signaling in osteoblasts integrates bone remodeling and energy metabolism. Cell 142:296-308.

Foresta C, Strapazzon G, De Toni L, Gianesello L, Bruttocao A, Scarda A, Plebani M, and Garolla A (2011) Androgens modulate osteocalcin release by human visceral adipose tissue. Clin Endocrinol (Oxf) 75:64-69.

Gardner LA, Naren AP, and Bahouth SW (2007) Assembly of an SAP97-AKAP79cAMP-dependent protein kinase scaffold at the type 1 PSD-95/DLG/ZO1 motif of the human $\beta_{1}$-adrenergic receptor generates a receptosome involved in receptor recycling and networking. J Biol Chem 282:5085-5099.
Iki M, Tamaki J, Fujita Y, Kouda K, Yura A, Kadowaki E, Sato Y, Moon JS, Tomioka $\mathrm{K}$, Okamoto N, et al. (2012) Serum undercarboxylated osteocalcin levels are inversely associated with glycemic status and insulin resistance in an elderly Japanese male population: Fujiwara-kyo Osteoporosis Risk in Men (FORMEN) Study. Osteoporos Int 23:761-770

Jacobsen SE, Ammendrup-Johnsen I, Jansen AM, Gether U, Madsen KL, and Bräuner-Osborne $\mathrm{H}$ (2017) The GPRC6A receptor displays constitutive internalization and sorting to the slow recycling pathway. J Biol Chem 292: 6910-6926.

Jørgensen S, Have CT, Underwood CR, Johansen LD, Wellendorph P, Gjesing AP, Jørgensen CV, Quan S, Rui G, Inoue A, et al. (2017) Genetic variations in the human G protein-coupled receptor class C, group 6, member A (GPRC6A) control cell surface expression and function. J Biol Chem 292:1524-1534.

Kapahi P, Chen D, Rogers AN, Katewa SD, Li PWL, Thomas EL, and Kockel L (2010) With TOR, less is more: a key role for the conserved nutrient-sensing TOR pathway in aging. Cell Metab 11:453-465.

Karsenty G and Olson EN (2016) Bone and muscle endocrine functions: unexpected paradigms of inter-organ communication. Cell 164:1248-1256.

Kinkade CW, Castillo-Martin M, Puzio-Kuter A, Yan J, Foster TH, Gao H, Sun Y, Ouyang X, Gerald WL, Cordon-Cardo C, et al. (2008) Targeting AKT/mTOR and ERK MAPK signaling inhibits hormone-refractory prostate cancer in a preclinical mouse model. J Clin Invest 118:3051-3064.

Ko E, Choi H, Kim B, Kim M, Park KN, Bae IH, Sung YK, Lee TR, Shin DW, and Bae YS (2014) Testosterone stimulates Duox1 activity through GPRC6A in skin keratinocytes. J Biol Chem 289:28835-28845.

Laplante M and Sabatini DM (2009) mTOR signaling at a glance. J Cell Sci 122: 3589-3594.

Laplante M and Sabatini DM (2012) mTOR signaling in growth control and disease. Cell 149:274-293.

Lee NK, Sowa H, Hinoi E, Ferron M, Ahn JD, Confavreux C, Dacquin R, Mee PJ, McKee MD, Jung DY, et al. (2007) Endocrine regulation of energy metabolism by the skeleton. Cell 130:456-469.

Lefkowitz RJ and Shenoy SK (2005) Transduction of receptor signals by $\beta$-arrestins. Science 308:512-517.

Li X, Nooh MM, and Bahouth SW (2013) Role of AKAP79/150 protein in $\beta 1$ adrenergic receptor trafficking and signaling in mammalian cells. J Biol Chem 288:33797-33812.

Long QZ, Du YF, Ding XY, Li X, Song WB, Yang Y, Zhang P, Zhou JP, and Liu XG (2012) Replication and fine mapping for association of the C2orf43, FOXP4, GPRC6A and RFX6 genes with prostate cancer in the Chinese population. PLoS One 7:e37866.

Mendoza MC, Er EE, and Blenis J (2011) The Ras-ERK and PI3K-mTOR pathways: cross-talk and compensation. Trends Biochem Sci 36:320-328.

Mera P, Laue K, Ferron M, Confavreux C, Wei J, Galán-Díez M, Lacampagne A Mitchell SJ, Mattison JA, Chen Y, et al. (2016) Osteocalcin signaling in myofibers is necessary and sufficient for optimum adaptation to exercise. Cell Metab 23: 1078-1092.

Nørskov-Lauritsen L, Jørgensen S, and Bräuner-Osborne H (2015) $N$-glycosylation and disulfide bonding affects GPRC6A receptor expression, function, and dimerization. FEBS Lett 589:588-597.

Otani T, Mizokami A, Hayashi Y, Gao J, Mori Y, Nakamura S, Takeuchi H, and Hirata M (2015) Signaling pathway for adiponectin expression in adipocytes by osteocalcin. Cell Signal 27:532-544.

Oury F, Ferron M, Huizhen W, Confavreux C, Xu L, Lacombe J, Srinivas P, Chamouni A, Lugani F, Lejeune $\mathrm{H}$, et al. (2013) Osteocalcin regulates murine and human fertility through a pancreas-bone-testis axis. J Clin Invest 123:2421-2433.

Pi M, Faber P, Ekema G, Jackson PD, Ting A, Wang N, Fontilla-Poole M, Mays RW, Brunden KR, Harrington JJ, et al. (2005) Identification of a novel extracellular cation-sensing G-protein-coupled receptor. $J$ Biol Chem 280: 40201-40209.

Pi M, Kapoor K, Wu Y, Ye R, Senogles SE, Nishimoto SK, Hwang DJ, Miller DD, Narayanan R, Smith JC, et al. (2015) Structural and functional evidence for testosterone activation of GPRC6A in peripheral tissues. Mol Endocrinol 29: 1759-1773.

Pi M, Kapoor K, Ye R, Hwang DJ, Miller DD, Smith JC, Baudry J, and Quarles LD (2018a) Computationally identified novel agonists for GPRC6A. PLoS One 13: e0195980.

Pi M, Kapoor K, Ye R, Nishimoto SK, Smith JC, Baudry J, and Quarles LD (2016a) Evidence for osteocalcin binding and activation of GPRC6A in $\beta$-cells. Endocrinology 157:1866-1880

Pi M, Kapoor K, Ye R, Smith JC, Baudry J, and Quarles LD (2018b) GPCR6A is a molecular target for the natural products gallate and EGCG in green tea. Mol Nutr Food Res 62:e1700770

Pi M, Nishimoto SK, and Quarles LD (2016b) GPRC6A: jack of all metabolism (or master of none). Mol Metab 6:185-193.

Pi M, Parrill AL, and Quarles LD (2010) GPRC6A mediates the non-genomic effects of steroids. J Biol Chem 285:39953-39964.

Pi M and Quarles LD (2012) GPRC6A regulates prostate cancer progression. Prostate 72:399-409.

Pi M, Wu Y, Lenchik NI, Gerling I, and Quarles LD (2012) GPRC6A mediates the effects of L-arginine on insulin secretion in mouse pancreatic islets. Endocrinology 153:4608-4615

Pi M, Wu Y, and Quarles LD (2011) GPRC6A mediates responses to osteocalcin in $\beta$-cells in vitro and pancreas in vivo. J Bone Miner Res 26:1680-1683.

Pittas AG, Harris SS, Eliades M, Stark P, and Dawson-Hughes B (2009) Association between serum osteocalcin and markers of metabolic phenotype. J Clin Endocrinol Metab 94:827-832.

Robertson MJ, Deane FM, Robinson PJ, and McCluskey A (2014) Synthesis of Dynole 34-2, Dynole 2-24 and Dyngo 4a for investigating dynamin GTPase. Nat Protoc 9: 851-870. 
Saxton RA and Sabatini DM (2017) mTOR signaling in growth, metabolism, and disease. Cell 168:960-976.

Takata R, Akamatsu S, Kubo M, Takahashi A, Hosono N, Kawaguchi T, Tsunoda T, Inazawa J, Kamatani N, Ogawa O, et al. (2010) Genome-wide association study identifies five new susceptibility loci for prostate cancer in the Japanese population. Nat Genet 42:751-754.

Thomsen ARB, Plouffe B, Cahill TJ III, Shukla AK, Tarrasch JT, Dosey AM, Kahsai AW, Strachan RT, Pani B, Mahoney JP, et al. (2016) GPCR-G protein$\beta$-arrestin super-complex mediates sustained $G$ protein signaling. Cell 166: 907-919.

Tomaszewski M, Eales J, Denniff M, Myers S, Chew GS, Nelson CP, Christofidou P, Desai A, Büsst C, Wojnar L, et al. (2015) Renal mechanisms of association between fibroblast growth factor 1 and blood pressure. J Am Soc Nephrol 26:3151-3160.

Tsvetanova NG and von Zastrow M (2014) Spatial encoding of cyclic AMP signaling specificity by GPCR endocytosis. Nat Chem Biol 10:1061-1065.

Wei J, Hanna T, Suda N, Karsenty G, and Ducy P (2014) Osteocalcin promotes $\beta$-cell proliferation during development and adulthood through Gprc6a. Diabetes 63: 1021-1031.
Wellendorph P and Bräuner-Osborne $\mathrm{H}$ (2009) Molecular basis for amino acid sensing by family C G-protein-coupled receptors. Br J Pharmacol 156:869-884.

White JP, Gao S, Puppa MJ, Sato S, Welle SL, and Carson JA (2013) Testosterone regulation of Akt/mTORC1/FoxO3a signaling in skeletal muscle. Mol Cell Endocrinol 365:174-186.

Ye R, Pi M, Cox JV, Nishimoto SK, and Quarles LD (2017) CRISPR/Cas9 targeting of GPRC6A suppresses prostate cancer tumorigenesis in a human xenograft model. $J$ Exp Clin Cancer Res 36:90.

Yoshii SR and Mizushima N (2017) Monitoring and measuring autophagy. Int J Mol Sci 18:1865.

Address correspondence to: Min Pi, Department of Medicine, University of Tennessee Health Science Center, 19 S Manassas St., Memphis, TN 38163. E-mail: mpi@uthsc.edu; or L. Darryl Quarles, Department of Medicine, University of Tennessee Health Science Center, 19 S Manassas St., Memphis, TN 38163. E-mail: dquarles@uthsc.edu 